\title{
Actividad catalítica de complejos de rodio (I) con ligandos bidentados a base de Fósforo en reacciones de hidroformilación de Canfeno
}

\section{Catalytic Activity of Rhodium (I) Complexes with Bidentate Phosphinic Ligands in the Hydroformylation of Camphene}

\author{
Liliana Montalvo*, Alberto Bolaños**, Armando Cabrera***
}

Recibido: Noviembre 30 de 2008

Aceptado: Mayo 3 de 2009

Correspondencia: Departamento de Química. Facultad de Ciencias Naturales, Exactas y de la Educación. Universidad del Cauca. Popayán, Colombia. Email: Imontalvo@unicauca.edu.co

\section{RESUMEN}

Se prepararon los complejos $\mathrm{RhCl}(\mathrm{dppe})$, $\mathrm{RhCl}(\mathrm{dppp})$ y $\mathrm{RhCl}(\mathrm{dppb})$ donde dppe = difenilfosfino etano, dppp = difenilfosfinopropano; $d p p b=$ difenilfosfinobutano. Estos complejos fueron usados como precursores catalíticos en la reacción de hidroformilación de canfeno a diferentes concentraciones y temperaturas. Se encuentra que el mejor precursor catalítico es el complejo de Rodio con el ligando dppp, al parecer el ángulo P-Rh-P es muy importante en la activación del sustrato.

Palabras clave: Catálisis homogénea, hidroformilación, canfeno, difosfinas, rodio.

\section{ABSTRACT}

We have prepared the complexes $R h C l(d p p e), R h C l(d p p p)$ and $R h C l(d p p b)$, where dppe = diphenylphosphinoethane; $d p p p=$ diphenylphosphinopropane; $d p p b=$ diphenylphosphinobutane. The above complexes were used as catalytic precursor in the hydroformylation of camphene at diferents concentrations and temperatures. It has found that the best catalytic precursor was the Rhodium complex with dppp as ligand it seems that the P-Rh-P angle is very important in substrate activation.

Keywords: Homogeneous catalysis, hydroformylation, camphene, diphosphines, rhodium.

\section{INTRODUCCIÓN}

os terpenos son una importante familia de productos naturales que se utiliza mucho en la industria del perfume. Desde que algunos de sus aldehídos han mostrado interesantes propiedades organolépticas, la hidroformilación de terpenos ha sido muy investigada y se dispone en la actualidad de patentes y literatura sobre el tema $[1,2,3,4]$.

La hidroformilación representa una ruta importante para obtener aldehídos y alcoholes que son difíciles de obtener por rutas sintéticas convencionales. En ladécada pasada estas reacciones catalíticas han tenido un especial atractivo y han sido muy utilizadasen química orgánica debido a que ha sido posible controlar en muchas ocasiones la quimio, regio y estereoselectividad de ellas por la presencia de ligandos auxiliares de fosforo [5,6]. Recientemente se ha mostrado que la quimio y regioselectividad de sistemas alílicos y propenilbencénicos catalizada por rodio depende fuertemente de las propiedades estéricas y electrónicas de los ligandos y se han desarrollado sistemas catalíticos para la síntesis selectiva de aldehídos lineales o ramificados [7]. Los aldehídos derivados de los monoterpenos han mostrado actividades biológicas y fitosanitarias y han sido usadas como saborizantes, productos de perfumería y farmacéuticos $[8,9]$.

Monoterpenos como el canfeno $[10,11,12,13]$ han sido hidroformilados en presencia de catalizadores de cobalto y rodio y con un sistema catalítico Platino - Estaño $[1,4,13]$ pero se dispone de poca información en la literatura sobre los productos estereoisoméricos alcanzados en esta reacción.

\footnotetext{
*Departamento de Química. Facultad de Ciencias Naturales, Exactas y de la Educación. Universidad del Cauca. Popayán, Colombia lmontalvo@unicauca.edu.co

** Departamento de Química, Facultad de Ciencias, Universidad del Valle. Apartado 25360, Cali, Colombia albolan@univalle.edu.co

*** Instituto de Química. Universidad Nacional Autónoma de México. arcaor1@servidor.unam.mx
} 
La separación entre las dos caras de los diasterómeros del canfeno no es nada fácil y los modelos de análisis molecular de ellas han mostrado que los dobles enlaces de las caras diastereotópicas del canfeno están muy impedidos y que hay muy poca diferencia estérica entre ellas.

La reacción general de hidroformilación del canfeno se observa en el esquema 1:
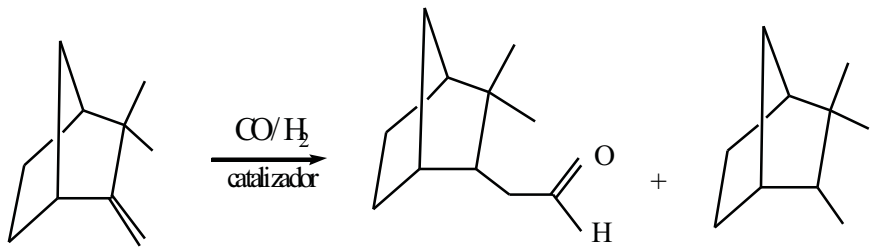

Esquema 1: Productos obtenidos en la reacción de hidroformilación del canfeno

\section{MATERIALES Y MÉTODOS}

Los complejos $\mathrm{RhCl}(\mathrm{dppe})$; $\mathrm{RhCl}(\mathrm{dppp})$ y $\mathrm{RhCl}(\mathrm{dppb})$ fueron sintetizados de la siguiente forma [14]: En un balón de $250 \mathrm{~mL}$ de 3 bocas, se adicionaron $60 \mathrm{ml}$ de acetona, la solución se purgó con nitrógeno durante 5 minutos, para eliminar el oxígeno presente y dependiendo del ligando se procedió así:

Tabla 1: Proporciones Estequiométricas utilizadas para obtener catalizadores

\begin{tabular}{|c|c|c|c|c|}
\hline $\begin{array}{c}\mathrm{RhCl}_{3} .3 \mathrm{H}_{2} \mathrm{O} \\
(\mathrm{mmol})\end{array}$ & $\begin{array}{c}\text { Ligando } \\
(\mathrm{mmol})\end{array}$ & Precipitado & Catalizador & Rendimiento \\
\hline 0.447 & 1.073 & amarillo & {$[\mathrm{RhCl}(\mathrm{dppe})]$} & $60 \%$ \\
\hline 0.3234 & 0.6973 & amarillo & {$[\mathrm{RhCl}(\mathrm{dppp})]$} & $64 \%$ \\
\hline 0.4982 & 0.9570 & amarillo & {$[\mathrm{RhCl}(\mathrm{dppb})]$} & $65 \%$ \\
\hline
\end{tabular}

La espectroscopia infrarrojo mostró las siguientes bandas:

Tabla 2: Asignación de bandas para los catalizadores $\mathrm{RhCl}(\mathrm{dppe}) ; \mathrm{RhCl}(\mathrm{dppp}) ; \mathrm{RhCl}(\mathrm{dppb})$

\begin{tabular}{|c|c|c|}
\hline Catalizador & Banda $\left(\mathrm{cm}^{-1}\right)$ & Asignación \\
\hline \multirow{2}{*}{ [RhCl(dppe)] } & 323.98 & $\mathrm{Rh}-\mathrm{Cl}$ \\
\hline & $296.8 \quad 257.4$ & Rh-P (cis) \\
\hline \multirow{2}{*}[\mathrm{RhCl}(\mathrm{dppp})]{} & 342.43 & $\mathrm{Rh}-\mathrm{Cl}$ \\
\hline & $296.98 \quad 230.45$ & Rh-P (cis) \\
\hline \multirow{2}{*}[\mathrm{RhCl}(\mathrm{dppb})]{} & 308.55 & $\mathrm{Rh}-\mathrm{Cl}$ \\
\hline & $285.41 \quad 267.09$ & Rh-P (cis) \\
\hline
\end{tabular}

Las reacciones de hidroformilación de canfeno con los catalizadores: $\mathrm{RhCl}(d p p e), \mathrm{RhCl}(\mathrm{dppp})$ y $\mathrm{RhCl}(\mathrm{dppb})$ se efectuaron en las proporciones moles sustrato : moles catalizador 1000:1 y 500:1. Estas reacciones se llevaron a cabo en un minirreactor Parr 4561 de $45 \mathrm{~mL}$. Para realizar estas reacciones el canfeno se diluyó en $10 \mathrm{mls}$ de tolueno y se le adicionó el respectivo catalizador en la cantidad necesaria de acuerdo a las proporciones antes mencionadas. El sistema se purgó con hidrógeno y monóxido de carbono. El reactor se programó a las $\mathrm{PH} 2 / \mathrm{CO}=20 / 20$ y $10 / 10$ bar y a las temperaturas de $120^{\circ} \mathrm{C}$ y $160^{\circ} \mathrm{C}$ con agitación constante. Se tomaron muestras a las $5,6,9,12,15,18$ y 24 horas de reacción con el objeto de monitorear la reacción.

\section{RESULTADOS Y DISCUSIÓN}

\section{Análisis de Espectros infrarrojo de los catalizadores:}

De acuerdo a los datos reportados en la literatura se observa que el cloro permanece coordinado al rodio ya que la banda $\mathrm{Rh}-\mathrm{Cl}$ continúa observándose en los espectros de los ligandos con ligeros desplazamientos debido al efecto de coordinación de los ligandos fosfina al rodio [15]. Por otra parte se observa la aparición de dos nuevas bandas reportadas en la literatura como características del enlace Rh-P [16].

\section{Reacciones de Hidroformilación de canfeno:}

El canfeno fue hidroformilado con los catalizadores $\mathrm{RhCl}(\mathrm{dppe}), \mathrm{RhCl}(\mathrm{dppp})$ y $\mathrm{RhCl}(\mathrm{dppb})$ en las proporciones moles sustrato : moles catalizador: 1000:1 y 500:1 a las temperaturas de $120^{\circ} \mathrm{C}$ y $160^{\circ} \mathrm{C}$ y $\mathrm{PH} 2 / \mathrm{CO}=20 / 20$ y $10 / 10$ bar. El análisis de estas reacciones fue hecho por cromatografía de gases y los compuestos obtenidos fueron caracterizados por GC-MS. Los productos obtenidos son: Aldehído y Canfano. Se tomaron blancos en las condiciones anotadas y se encontró que el canfeno no sufre ninguna reacción. Los datos obtenidos para el GC-MS son:

2-(biciclo[2,2,1]2-heptanil)acetaldehído: 166/14 (M+); 122/75. Canfano: 2,2,3- trimetilbiciclo2,2,1 heptano) 138/20 $(\mathrm{M}+) ; 123 / 15 ; 109 / 80 ; 95 / 100 ; 55 / 40$.

a) Catalizador $\mathrm{RhCl}(\mathrm{dppb})$ :

Tabla 3: Hidroformilación canfeno : RhCl(dppb) 1000:1, $\mathrm{PH} 2 / \mathrm{CO}=20 / 20$ bar.

\begin{tabular}{|c|c|c|c|c|c|c|}
\hline & \multicolumn{2}{|c|}{$\%$ canfeno } & \multicolumn{2}{|c|}{$\%$ aldehído } & \multicolumn{2}{c|}{$\%$ canfano } \\
\hline $\begin{array}{c}\text { Tiempo } \\
\text { (horas) }\end{array}$ & $160{ }^{\circ} \mathrm{C}$ & $120^{\circ} \mathrm{C}$ & $160^{\circ} \mathrm{C}$ & $120^{\circ} \mathrm{C}$ & $160{ }^{\circ} \mathrm{C}$ & $120^{\circ} \mathrm{C}$ \\
\hline 0 & 100 & 100 & 0 & 0 & 0 & 0 \\
\hline 6 & 41.8 & 49.1 & 54.1 & 50.9 & 4.13 & 0 \\
\hline 9 & 27.7 & 45.3 & 53.1 & 54.7 & 19.3 & 0 \\
\hline 12 & & 21.8 & & 72.8 & & 5.4 \\
\hline 15 & 11.4 & 10.8 & 71.1 & 84.2 & 17.4 & 5.0 \\
\hline 18 & 6.2 & 7.0 & 79.0 & 88.4 & 14.8 & 5.6 \\
\hline 24 & 6.2 & 5.4 & 79.1 & 91.0 & 14.7 & 3.5 \\
\hline
\end{tabular}



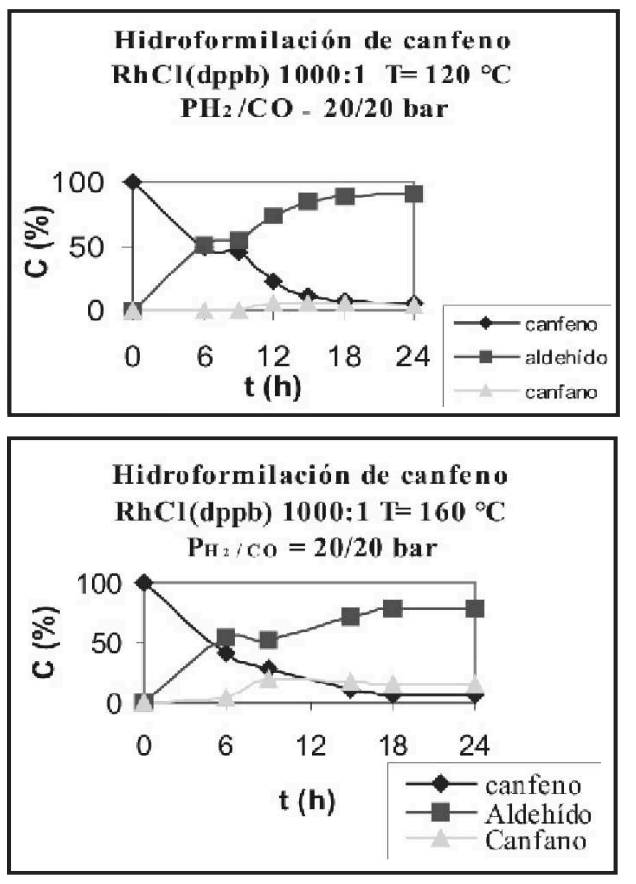

Cuando se realiza la reacción de hidroformilación de canfeno con este catalizador, a las condiciones dadas, se observa que el canfeno se consume casi completamente a las 24 horas de reacción, independiente de la temperatura, pero a menor temperatura la reacción procede casi en su totalidad hacia la formación de aldehído, mientras que a mayor temperatura se favorece la reacción indeseada, la hidrogenación.

Tabla 4: Hidroformilación canfeno : $\mathrm{RhCl}(\mathrm{dppb}) \mathrm{T}=120^{\circ} \mathrm{C}$, $\mathrm{PH} 2 / \mathrm{CO}=10 / 10$ bar.

\begin{tabular}{|c|c|c|c|c|c|c|}
\hline & \multicolumn{2}{|c|}{$\%$ canfeno } & \multicolumn{2}{c|}{$\%$ aldehído } & \multicolumn{2}{c|}{$\%$ canfano } \\
\hline $\begin{array}{c}\text { Tiempo } \\
\text { (horas) }\end{array}$ & $1000: 1$ & $500: 1$ & $1000: 1$ & $500: 1$ & $1000: 1$ & $500: 1$ \\
\hline 0 & 100 & 100 & 0 & 0 & 0 & 0 \\
\hline 5 & 100 & 81 & 0 & 19 & 0 & 0 \\
\hline 9 & 83.3 & 80.7 & 16.7 & 19.3 & 0 & 0 \\
\hline 13 & 76.2 & 60.6 & 20.7 & 36.4 & 3 & 3.1 \\
\hline 15 & 67.2 & 57.8 & 26.1 & 37.4 & 6.6 & 4.8 \\
\hline 18 & 57.2 & & 42.8 & & 0 & \\
\hline 25 & 47.4 & 48.7 & 50.1 & 51.3 & 1.46 & 0 \\
\hline
\end{tabular}

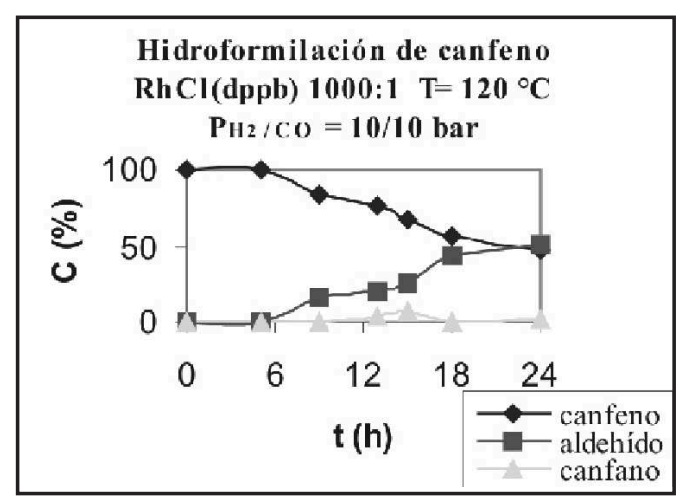

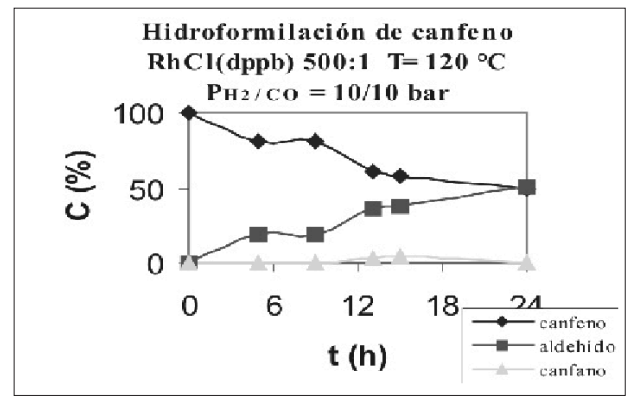

La reacción de hidroformilación de canfeno con este catalizador a la temperatura de $120^{\circ} \mathrm{C}$, sustrato:catalizador $1000: 1$ y $\mathrm{PH} 2 / \mathrm{CO}=20 / 20$ bar y $10 / 10$ bar muestra que a una mayor relación $\mathrm{PH} 2 / \mathrm{CO}$ se produce una mayor cantidad de aldehído, mientras que a una menor relación $\mathrm{PH} 2 / \mathrm{CO}$ permanece sin reaccionar un poco menos de un $50 \%$.

Al varíar las proporciones sustrato: catalizador de 1000:1 a 500:1, a la temperatura de $120^{\circ} \mathrm{C}$ y $\mathrm{PH} 2 / \mathrm{CO}=10 / 10$ bar, se observa que en ambas reacciones casi el $50 \%$ de canfeno permanece sin reaccionar, sugiriendo que ésta no es una buena $\mathrm{PH} 2 / \mathrm{CO}$ para hidroformilar este sustrato.

\section{b) Promotor RhCl(dppp):}

Tabla 5: Hidroformilación canfeno : $\mathrm{RhCl}(\mathrm{dppp}) \mathrm{T}=120^{\circ} \mathrm{C}$, $\mathrm{PH} 2 / \mathrm{CO}=10 / 10$ bar.

\begin{tabular}{|c|c|c|c|c|c|c|}
\hline & \multicolumn{2}{|c|}{$\%$ canfeno } & \multicolumn{2}{c|}{$\%$ aldehído } & \multicolumn{2}{c|}{$\%$ canfano } \\
\hline $\begin{array}{c}\text { Tiempo } \\
\text { (horas) }\end{array}$ & $1000: 1$ & $500: 1$ & $1000: 1$ & $500: 1$ & $1000: 1$ & $500: 1$ \\
\hline 0 & 100 & 100 & 0 & 0 & 0 & 0 \\
\hline 6 & 80.87 & 100 & 19.13 & 0 & 0 & 0 \\
\hline 9 & 77.8 & 99 & 22.1 & 1 & 0 & 0 \\
\hline 13 & 70 & & 30 & & 0 & \\
\hline 15 & 63.4 & 83.43 & 34.34 & 16.57 & 1.98 & 0 \\
\hline 18 & & 75.8 & & 24.2 & & 0 \\
\hline 20 & 42.36 & 52.7 & 54 & 47.2 & 3.8 & 0 \\
\hline
\end{tabular}
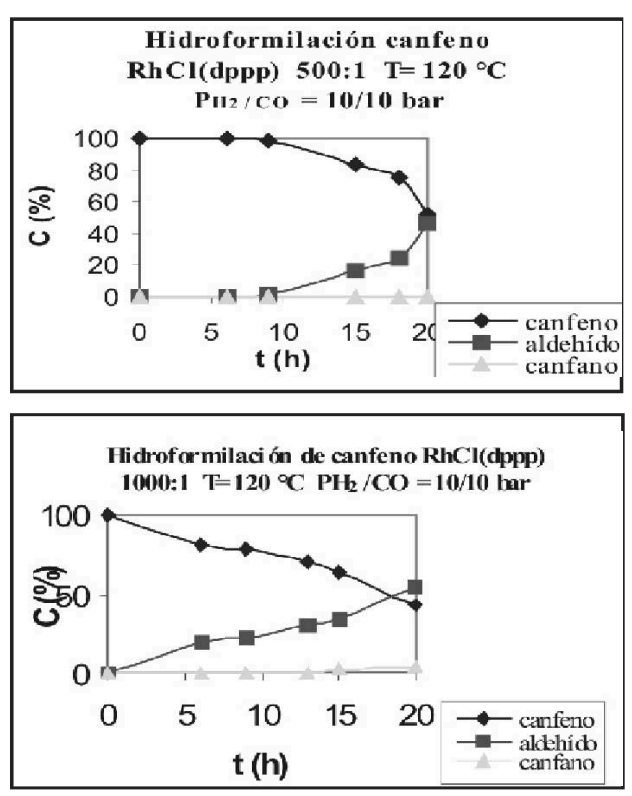
Tabla 6: Hidroformilación canfeno : $\mathrm{RhCl}(\mathrm{dppp}) \mathrm{T}=120^{\circ} \mathrm{C}$, $\mathrm{PH} 2 / \mathrm{CO}=20 / 20$ bar

\begin{tabular}{|c|c|c|c|c|c|c|}
\hline & \multicolumn{2}{|c|}{$\%$ canfeno } & \multicolumn{2}{c|}{$\%$ aldehído } & \multicolumn{2}{c|}{$\%$ canfano } \\
\hline $\begin{array}{c}\text { Tiempo } \\
\text { (horas) }\end{array}$ & $1000: 1$ & $500: 1$ & $1000: 1$ & $500: 1$ & $1000: 1$ & $500: 1$ \\
\hline 0 & 100 & 100 & 0 & 0 & 0 & 0 \\
\hline 5 & 71.5 & 58.6 & 27 & 41.4 & 1.6 & 0 \\
\hline 9 & & 22.1 & & 78.1 & & 0 \\
\hline 14 & & 7.64 & & 88.5 & & 3.9 \\
\hline 15 & 61 & & 39 & & 0 & \\
\hline 18 & 34.9 & 6.41 & 65.1 & 91 & 0 & 2.6 \\
\hline 24 & 20.1 & & 78.5 & & 1.3 & \\
\hline
\end{tabular}
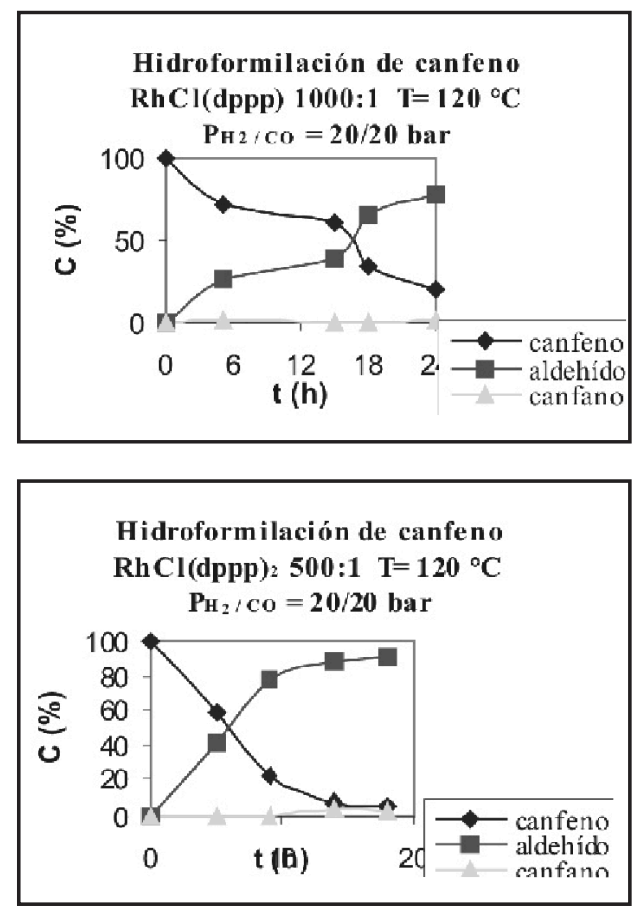

La reacción de hidroformilación de canfeno con este catalizador a la temperatura de $120^{\circ} \mathrm{C}$, sustrato:catalizador 1000:1, 500:1 y PH2/CO = 10/10 bar no presenta cambios significativos en los resultados, aunque a una mayor proporción de catalizador no se observa producto de hidrogenación. Al varíar la $\mathrm{PH} 2 / \mathrm{CO}$ a 20/20 bar se observa que a una mayor concentración de catalizador la reacción procede en un $90 \%$ a la formación de aldehído, sugiriendo que esta presión es la más adecuada en esta reacción.

Cuando se comparan los resultados obtenidos en la hidroformilación de canfeno con ambos catalizadores se observa que la mejor relación es la de $\mathrm{PH} 2 / \mathrm{CO}=20 / 20$ bar, pero que con el $\mathrm{RhCl}(\mathrm{dppb})$ se requiere una menor concentración de catalizador para que la reacción suceda aproximadamente en un $90 \%$.

\section{c) Promotor RhCl(dppe):}

Con este catalizador no se presenta producto de hidroformilación.

En todas las reacciones efectuadas se observa que a una mayor concentración de catalizador se favorece la reacción de hidrogenación, lo que sugiere un cambio en la selectividad de la reacción. Se ha reportado que cuando la concentración de rodio es alta, se forma una especie de rodio carbonilo desconocida a partir del hidruro de rodio, que es poco activa para la hidroformilación [17].

La literatura reporta que el "ángulo mordida" de las difosfinas ejerce una influencia significativa en la velocidad de reacción, encontrándose que los "ángulos mordida" mayores favorecen la formación de aldehídos [18]. Los "ángulo mordida" P-Rh-P son: $85^{\circ}$ para Rh-dppe; $91^{\circ}$ para Rh-dppp y $95^{\circ}$ para Rh-dppb, formándose anillos de cinco, seis y siete miembros [19].

\section{CONCLUSIONES}

El espectro infrarrojo de los complejos $\mathrm{RhCl}(\mathrm{dppe})$; $\mathrm{RhCl}(\mathrm{dppp})$ y $\mathrm{RhCl}(\mathrm{dppb})$ muestra que se ha producido la coordinación del ligando bidentado de fósforo al rodio.

En las reacciones efectuadas se observa la tendencia de que las reacciones suceden mejor a una mayor $\mathrm{PH} 2 / \mathrm{CO}$, evidenciándose una formación casi completa de aldehído. Estas reacciones son independientes de la concentración de catalizador, lo que sugiere que éste es un factor determinante.

Las reacciones efectuadas con el catalizador $\mathrm{RhCl}(\mathrm{dppe})$ no presentan ningún producto de reacción, lo que confirma la estabilidad de los anillos de cinco miembros.

En todas las reacciones efectuadas se observa que a una mayor concentración de catalizador se acelera la reacción de hidroformilación y se inhibe por completo la reacción de hidrogenación.

El aumento de temperatura favorece la reacción indeseada de hidrogenación, se sugiere que el catalizador puede cambiar de selectividad con este factor.

\section{AGRADECIMIENTOS}

Los autores agradecen a la Universidad del Cauca, a la Universidad del Valle y a la Universidad Nacional Autónoma de México por la colaboración prestada para la realización de esta investigación. 


\section{BIBLIOGRAFÍA}

(1) Gusevskaya EV, dos Santos EN, Augusti R, Dias AO, Foca CM. Platinum/Tin catalyzed hydroformylation of naturally occurring monoterpenes. J. Mol. Catal. A 2000, 152, 15-24.

(2) Dias AO, Augusti R, dos Santos EN, Gusevskaya E. Convenient one-pot syntheses of 4,8-dimethyl-biyclo[3,3,1]-non-7-en-2ol. Tetrahedron Lett. 199738, 41-4.

(3) Barros H, Ospina ML, Arguello E, Rocha WE, Gusevskaya EV, dos Santos EN. Rhodium catalyzed hydroformylation of $\beta$ pinene and camphene: effect of phosphorous ligands and reaction conditions on diastereoselectivity. J. Organomet. Chem., 2003, 671, 150-7.

(4) Foca CM, dos Santos EN, Gusevskaya EV. Diastereoselective hydroformylation of camphene catalyzed by platinum/tin complexes. J. Mol. Catal. A, 2002, 185, 17-23.

(5) Van Leeuwen PWNM, Claver C. Editors, Rhodium Catalyzed Hydroformylation, Kluwer Academic Publisher, Dordrecht, 2000, p.87-102 ISBN 07923-6551.

(6) Breit B, Seiche W, Recent Advances in Chemo-Regio-and Stereoselective Hydroformylation. Synthesis, 2001, 1-36.

(7) da Silva AC, de Oliveira KCB, Gusevskaya EV, dos Santos EN, Rhodium catalyzed hydroformylation of allylbenzenes and propenylbenzenes: effect of phosphine and diphosphine ligands on chemo- and regioselectivity. J. Mol. Catal. 2002, 179, 133-141.

(8) Erman WE, Chemistry of the Monoterpenes. An Encyclopedic Handbook, Marcel Dekker, New York 1985.

(9) Chalk AJ, Rylander PN, Greenfield H, Augustine RL. Editors, Catalysis of Organic Reactions 22, Marcel Dekker, New York, 1988.

(10) Gusevskaya EV. Organometallic catalysis: Some contributions to organic synthesis. Quim. Nova, 2003, 26 (2), 242-8.

(11) Hagen J, Bruns K, Henkel K, Patent DE 2849742, 1980.

(12) Yuan K, Yuanqi Y, Cuihua F. 1989, 3, 262 Chem. Abstr, 114:6831 r.

(13) Kollár L, Bódi G. Hydroformylation of Terpenes and Sesquiterpenes. Chirality 1995, 1, 121-6.

(14) Montalvo L, Bolaños A, Cabrera A. Síntesis de catalizadores de rodio (I) con ligandos bidentados basados en fósforo y su actividad catalítica en reacciones de hidroformilación de 1,5- ciclooctadieno. El hombre y la maquina, 2006, 27 (XVIII), 8693.

(15)Jennings MA, Wojcicki, Thyocianato complexes of rhodium (I). Inorg. Chem., 1967, 6, 1854-9.

(16) Brown J, Kent A. Structural characterization in solution of intermediates in rhodium-catalyzed hydroformylation and their interconversion pathways. J. Chem. Soc., Perkin Trans., 1987, 2, 1597-1607.

(17) Bronger RPJ, Kamer PCJ, Van Leeuwen PWNM, Influence of the Bite angle on the Hydroformylation of Internal Olefins to linear Aldehydes. Organometallic, 2003, 22 (25), 5358-69.

(18)Casey C.P, Petrovich LM. Chelating diphosphine rhodium catalyzed deuterioformylation of 1-hexene: Control of regiochemistry by the kinetic ratio of alkyl rhodium species formed by hydride addition to complexed alkene. J.Am.Chem.Soc., 1995, 117, 6007-14.

(19) Rocha Gonsalvez A, Bayón JC, Pereira MM, Serra MES, Pereira JPR. Assymmetric transfer hydrogenation of acrylic acids catalyzed by rhodium (I) complexes of diphosphine ligands. J. Organomet. Chem., 1998, 553, 199-204. 